\title{
Glycinergic Inhibition Contributes to the Generation of Rostral Scratch Motor Patterns in the Turtle Spinal Cord
}

\author{
Scott N. Currie and Steven Lee \\ Department of Neuroscience, University of California, Riverside, California 92521
}

Cutaneous stimulation within the rostral scratch receptive field in a low spinal-immobilized turtle elicits a fictive rostral scratch reflex characterized by robust rhythmic motor output from ipsilateral hindlimb muscle nerves and weaker, alternating motor discharge in contralateral nerves. Simultaneous bilateral stimulation elicits bilateral rostral scratch motor patterns in which activity on the right and left sides alternates.

We investigated the role of glycinergic inhibition in the generation and coordination of fictive rostral scratch motor patterns. Glycine (2 or $5 \mathrm{~mm}$ ) and strychnine (5-50 $\mu \mathrm{M})$, a glycine antagonist, were superfused over the anterior spinal hindlimb enlargement while fictive rostral scratch motor output was recorded bilaterally from hindlimb muscle nerves in the form of electroneurograms (ENGs). Although glycine reduced rostral scratch burst frequencies, strychnine tended to increase burst frequency. Strychnine also changed the shape of hip flexor ENG bursts, resulting in more abrupt burst onsets, indicating an earlier recruitment of motor neurons with large ENG spikes. During bilateral stimulation, strychnine increased the variability of interlimb phase values (left vs right hip flexor bursts) but did not abolish right-left alternation.

These results indicate that glycinergic neurons in or near the anterior hindlimb enlargement contribute to the overall timing of the rostral scratch rhythm and to the recruitment timing of individual hip flexor motor neurons within each scratch burst. Our data also indicate that glycinergic mechanisms contribute to, but are not critically important for, maintaining an alternating interlimb coordination during bilateral scratch motor patterns.

Key words: turtle; spinal; scratch reflex; central pattern generator; inhibition; strychnine
The turtle spinal cord is capable of performing a complex sensorimotor transformation, termed a scratch reflex, in which a hindlimb is directed to approach and rhythmically rub the site of a cutaneous stimulus on the body surface (Stein, 1989). The neural calculations required for this task can be performed in the absence of descending signals from the brain and without movement-related sensory feedback from the limb. Low spinalimmobilized turtles display three forms of the fictive scratch reflex in hindlimb muscle nerves in response to tactile stimulation within different regions of the body surface (Robertson et al., 1985). Stimulation of sites on the "shell-bridge," anterior to the hindlimb, evokes the rostral form of the scratch. Fictive rostral scratch motor patterns are characterized by (1) rhythmic alternation between hip flexor and hip extensor ENG bursts and (2) monoarticular knee extensor (femorotibialis) ENG discharge during the late hip flexor phase of the scratch cycle.

Intracellular recordings from turtle hindlimb motor neurons suggested that synaptic inhibition might have a significant role in shaping rostral scratch motor output (Robertson and Stein, 1988). Those authors demonstrated two phases of inhibition onto hip flexor motor neurons during the rostral scratch. One phase of inhibition occurred during the activation of the antagonist hip extensor motor pool, while the hip flexor motor pool was quiescent (off-cycle inhibition). A second phase of inhibition occurred during the early portion of the hip flexor ENG burst and was related to the recruitment-timing of motor neurons within the

Received Oct. 31, 1996; revised Feb. 11, 1997; accepted Feb. 14, 1997.

This research was supported by National Science Foundation Grant IBN-9308804 to S.N.C. We thank A. Berkowitz and B. G. Stanley for their editorial assistance.

Correspondence should be addressed to Dr. Scott N. Currie, Department of Neuroscience, University of California, Riverside CA 92521.

Copyright (C) 1997 Society for Neuroscience 0270-6474/97/173322-12\$05.00/0 burst (on-cycle inhibition). This on-cycle inhibition delayed the onset of spiking in some hip flexor motor neurons and contributed to the slow, ramp-like onset of the whole-nerve ENG burst. Knee extensor (femorotibialis) motor neurons exhibited a similar pattern of inhibition during the rostral scratch, including hyperpolarization during the hip extensor phase and inhibitory input during the early hip flexor phase that delayed firing onset until the latter half of the hip flexor burst.

Very little is currently known about the identity of inhibitory neurotransmitter systems in vertebrate scratch circuitry. Previous researchers (Creed et al., 1932; Hart, 1971) observed only that intravenous injection of strychnine, a glycine receptor antagonist (Goodman-Gilman et al., 1991), "facilitated" scratching, flexion withdrawal, and various other reflex movements in low-spinal mammals. In the present study, we investigated the role of glycinergic inhibition in the generation and coordination of fictive rostral scratch motor patterns. Glycine or strychnine were superfused over spinal cord segments in and near the anterior hindlimb enlargement (D7-D8 or D7-D10) in low-spinal turtles while unilateral or bilateral scratch motor output from hindlimb nerves was recorded. Our results support the hypothesis that glycinergic neurons contribute to the overall timing of the rostral scratch rhythm and to the recruitment timing of hip flexor motor neurons within each scratch cycle. In addition, we provide evidence that glycinergic mechanisms help to stabilize right-left coordination of bilateral scratch motor output but are not required to maintain the normal alternating relationship.

These data were published previously in abstract form (Currie and Lee, 1995).

\section{MATERIALS AND METHODS}

Red-eared turtles ( $n=14$; Kons Scientific, Germantown, WI), Trachemys scripta elegans, weighing 470-650 gm, were placed in crushed ice for $2 \mathrm{hr}$ 


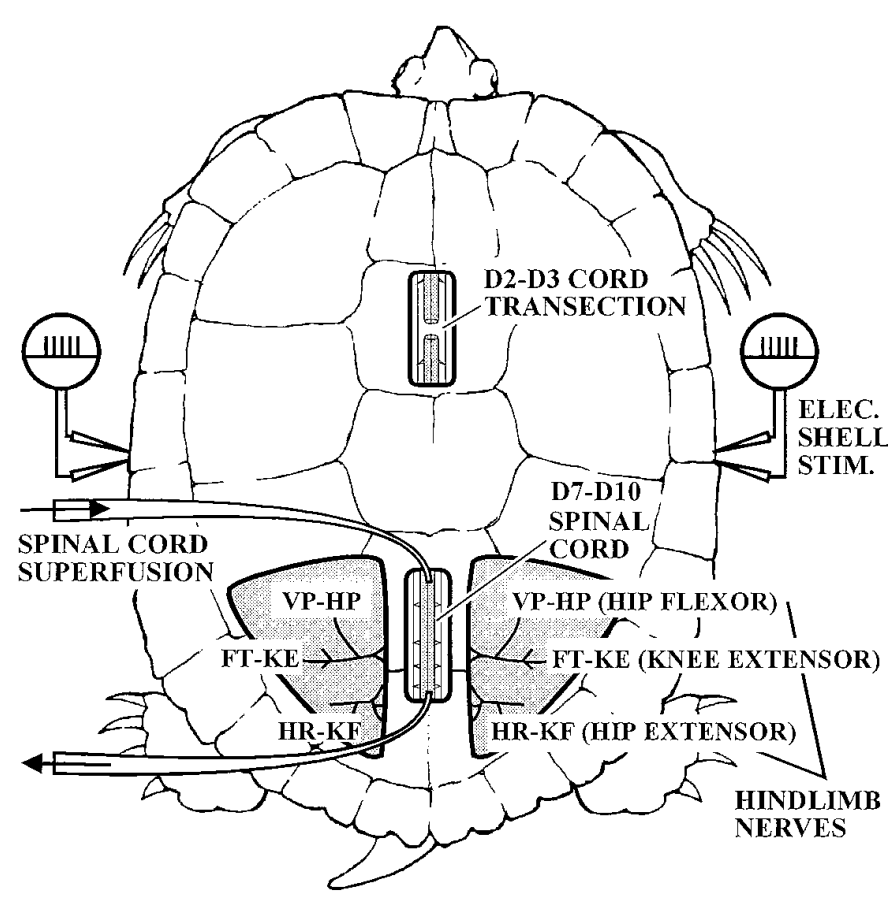

Figure 1. Dorsal view of the experimental preparation, showing segments D7-D10 of the spinal cord hindlimb enlargement exposed for drug superfusion. The exposed segments were stripped of all meninges on their dorsal and dorsolateral surfaces to facilitate diffusional exchange between the superfusate and the spinal tissue. Fictive rostral scratch motor patterns were elicited by electrical or mechanical stimulation of the shell in the right and/or left rostral scratch receptive field(s) and recorded bilaterally from hindlimb muscle nerves. Recordings were obtained from nerves innervating a monoarticular KE (FT-KE), an HF (VP-HP), and an HE (HR-KF) muscle. See Materials and Methods for a complete description of the preparation and surgical procedures.

before surgery to induce hypothermic analgesia (Maxwell, 1979; Marcus, 1981). Turtles were maintained partially immersed in crushed ice during all surgical procedures. The first surgical procedure was complete transection of the spinal cord just posterior to the forelimb enlargement, between spinal segments D2 and D3 (D2 = the second postcervical segment; Zangerl, 1969).

\section{Surgical procedures}

Hindlimb muscle nerves were prepared bilaterally for ENG recording (Fig. 1). The FT-KE nerve innervates triceps femoris pars femorotibia- lis, a monoarticular knee extensor muscle. VP-HP innervates puboischiofemoralis internus, pars anteroventralis, a hip flexor (protractor) muscle. HR-KF innervates several bifunctional hip extensor (retractor), knee flexor muscles of the flexor tibialis group. These nerves and the muscles they innervate have been described previously (Robertson et al., 1985). In twelve turtles, we prepared FT-KE, VP-HP, and HR-KF bilaterally; in two turtles, we prepared FT-KE and VP-HP bilaterally. Each nerve was freed from surrounding tissues, tied with surgical thread near its muscle insertion, and then cut distal to the tie. Hereafter in the text, FT-KE is referred to as the knee extensor (KE) nerve, VP-HP as the hip flexor (HF) nerve, and HR-KF as the hip extensor (HE) nerve.

We exposed two or four segments of spinal cord at the anterior side of the hindlimb enlargement by drilling a midline channel through the dorsal carapace and performing a dorsal laminectomy (Fig. 1). The turtle hindlimb enlargement consists of three dorsal segments (D8, D9, D10) and two sacral segments (S1, S2). We exposed either segments D7-D8 (Experiments 1, 3, 5) or D7-D10 (Experiments 2, 4, 6-14). In three of the preparations with D7-D10 exposures, we transected the spinal cord within the exposed region, at the posterior end of either the D9 (Experiment 12) or D10 (Experiments 13, 14) segment; this eliminated scratch motor pattern generating circuitry located in posterior segments of the hindlimb enlargement (segments S1-S2), outside of the drug-soak region. Removal of these segments does not significantly alter the fictive rostral scratch motor pattern (Mortin and Stein, 1989). All meninges were stripped from the dorsal and dorsolateral surfaces of the exposed spinal segments. Plugs of Gelfoam surgical sponge were inserted into the vertebral canal between the bone and the dorsal surface of the cord at the anterior and posterior ends of the region of the laminectomy; these plugs reduced the bulk flow of drugs out of the exposed region.

\section{ENG recordings}

After surgery was complete, preparations were allowed to warm up to room temperature and then were immobilized with an intramuscular injection of gallamine triethiodide $(6 \mathrm{mg} / \mathrm{kg}$ body weight $)$. The trachea was intubated, and artificial respiration was used throughout the experiment. The skin was kept moist with turtle saline. Rings of warm dental wax were formed around the holes in the dorsal carapace over the dissected hindlimb nerves and the exposed spinal cord, allowed to cool and harden, and glued in place with cyanoacrylate adhesive. The dissected hindlimb nerves were then strung out for recording by securing their attached threads to the lips of the wax well. Bipolar hook electrodes (100 $\mu \mathrm{m}$ diameter, silver) were used to record from the nerves in a pool of mineral oil. Electrical signals were amplified (bandpass $100 \mathrm{~Hz}-1 \mathrm{kHz}$ ), digitized by a PCM video adapter (Vetter, Rebersburg, PA), and stored along with a voice channel and stimulus marker on videotape (bandpass DC-3.5 kHz). Data were later redigitized off-line at $2 \mathrm{kHz}$ on a 486 computer, formatted using analysis (Run Technologies, Laguna Hills, CA) and graphics (Corel, Ottawa, Canada) software, and plotted on a laser printer.

\section{Table 1. Strychnine increased rostral scratch motor burst frequencies}

\begin{tabular}{|c|c|c|c|c|c|}
\hline Experiment & $\begin{array}{l}\text { Cord } \\
\text { exposure }\end{array}$ & Stimulus & $\begin{array}{l}\text { Control } \\
\text { average (SD) }\end{array}$ & $\begin{array}{l}\text { Strychnine } \\
\text { average (SD) }\end{array}$ & $\begin{array}{l}\text { Wash } \\
\text { average (SD) }\end{array}$ \\
\hline 1 & D7-D8 & Right & $100.0(14.0)$ & $115.0(34.3)^{\mathrm{NS}}$ & $99.2(27.3)^{\mathrm{NS}}$ \\
\hline 1 & D7-D8 & Left & $100.0(16.6)$ & $121.6(19.0)^{\S}$ & $124.5(21.8)^{\mathrm{NS}}$ \\
\hline 2 & D7-D10 & Right & $100.0(15.8)$ & $123.2(46.8)^{\mathrm{NS}}$ & $107.4(30.4)^{\mathrm{NS}}$ \\
\hline 2 & D7-D10 & Left & $100.0(8.4)$ & $142.5(28.9)^{*}$ & $88.4(9.8)^{*}$ \\
\hline 8 & D7-D10 & Right & $100.0(11.8)$ & $148.7(40.8)^{*}$ & $102.9(14.6)^{*}$ \\
\hline 9 & D7-D10 & Right & $100.0(8.8)$ & $138.7(15.5)^{*}$ & $111.7(12.5)^{\S}$ \\
\hline 10 & D7-D10 & Right & $100.0(21.3)$ & $118.2(19.5)^{\ddagger}$ & $99.0(19.8)^{\mathrm{NS}}$ \\
\hline 10 & D7-D10 & Left & $100.0(11.4)$ & $175.0(27.8)^{*}$ & $108.7(16.6)^{\S}$ \\
\hline 11 & D7-D10 & Right & $100.0(38.0)$ & $194.7(56.1)^{\S}$ & $123.0(33.7)^{\S}$ \\
\hline
\end{tabular}

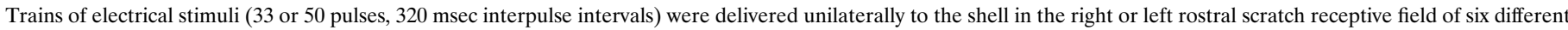

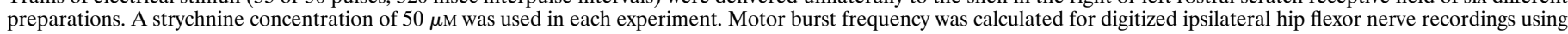

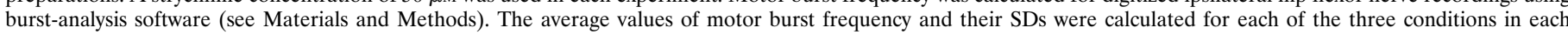

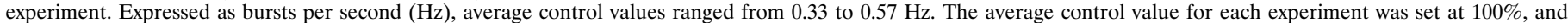

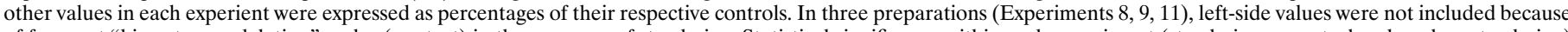

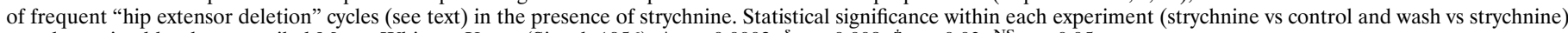
was determined by the one-tailed Mann-Whitney $U$ test (Siegel, 1956). ${ }^{*} p<0.0002 ;{ }^{\circledR} p<0.008 ;{ }^{\ddagger} p<0.03 ;{ }^{\mathrm{NS}} p>0.05$. 


\section{A CONTROL}

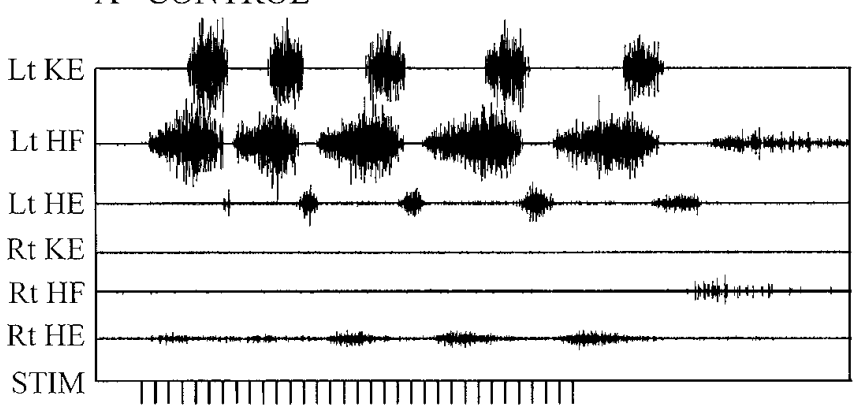

B STRYCHNINE

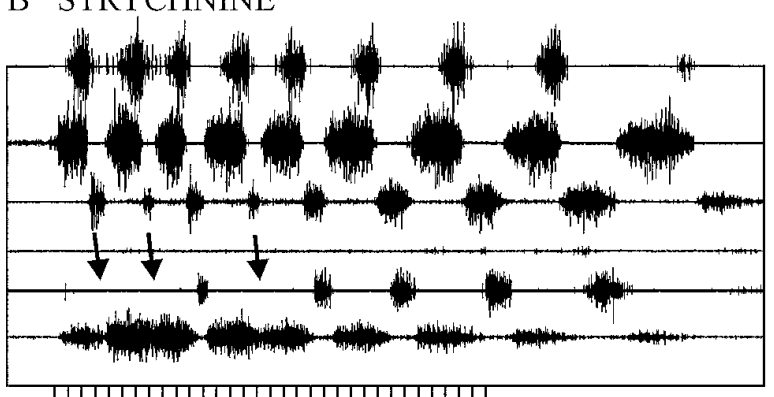

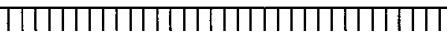

C WASH

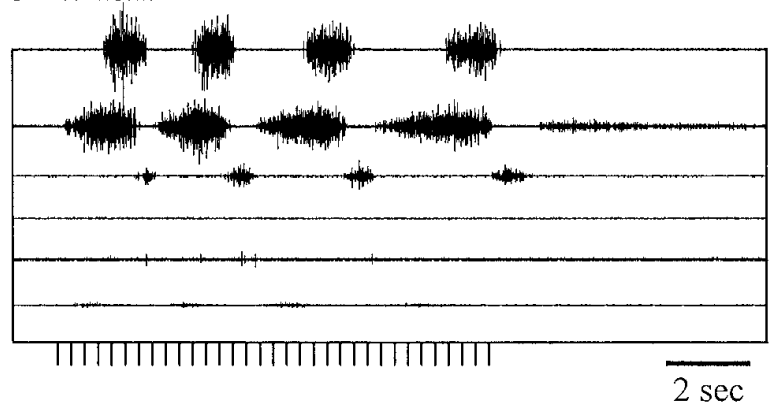

Figure 2. Strychnine increased the burst frequency of fictive rostral scratch motor patterns and increased the amplitude of contralateral motor output during unilateral stimulation of a site (SP2.5) in the left rostral scratch receptive field. Motor output was recorded bilaterally from hindlimb muscle nerves in Experiment 10, a D3-end preparation. D3-end preparations had intact spinal cords posterior to the D2-D3 transection site. Stimulation consisted of trains of 33 electrical pulses $(10 \mathrm{~V}, 1 \mathrm{msec}$ pulses) delivered at $320 \mathrm{msec}$ interpulse intervals. Recordings were obtained from a knee extensor $(K E)$, a hip flexor $(H F)$, and a hip extensor $(H E)$ muscle nerve. $A$, Control response obtained while spinal segments D7-D10 of the anterior hindlimb enlargement were bathed in normal saline. $B$, After $1.25 \mathrm{hr}$, superfusion of the exposed spinal segments with 50 $\mu \mathrm{M}$ strychnine. Note the appearance of right-side cHF bursts and the increased amplitude of right HE bursts compared with the control. Arrows in $B$ indicate the timing of cHF deletions: cycles that lacked cHF bursts. $C$, After $7 \mathrm{hr}+25 \mathrm{~min}$ wash with normal saline.

\section{Stimulation}

We used either mechanical or electrical stimulation of the shell to evoke fictive rostral scratch reflexes. Scratch episodes were always separated by rest periods of $\geq 2 \mathrm{~min}$. Mechanical stimulation was applied by gently rubbing a site on the shell with a fire-polished glass probe; the probe was mounted on a hand-held force transducer (Astro-Med, Warick, RI) to record the force and timing of the stimulus. Rubs with the glass probe were applied with a force of $0.2-1.4 \mathrm{~N}$ (Newtons) and lasted 10-15 sec. Electrical stimulation was applied either unilaterally or bilaterally to sites in the rostral scratch receptive field via pin electrodes inserted into the shell epidermis 2-3 mm apart (Currie and Stein, 1990). Pulses of 10-20 $\mathrm{V}$ amplitude and $1 \mathrm{msec}$ duration were delivered in 33- or 50-pulse trains with an interpulse interval of $320 \mathrm{msec}$. For experiments in which bilateral
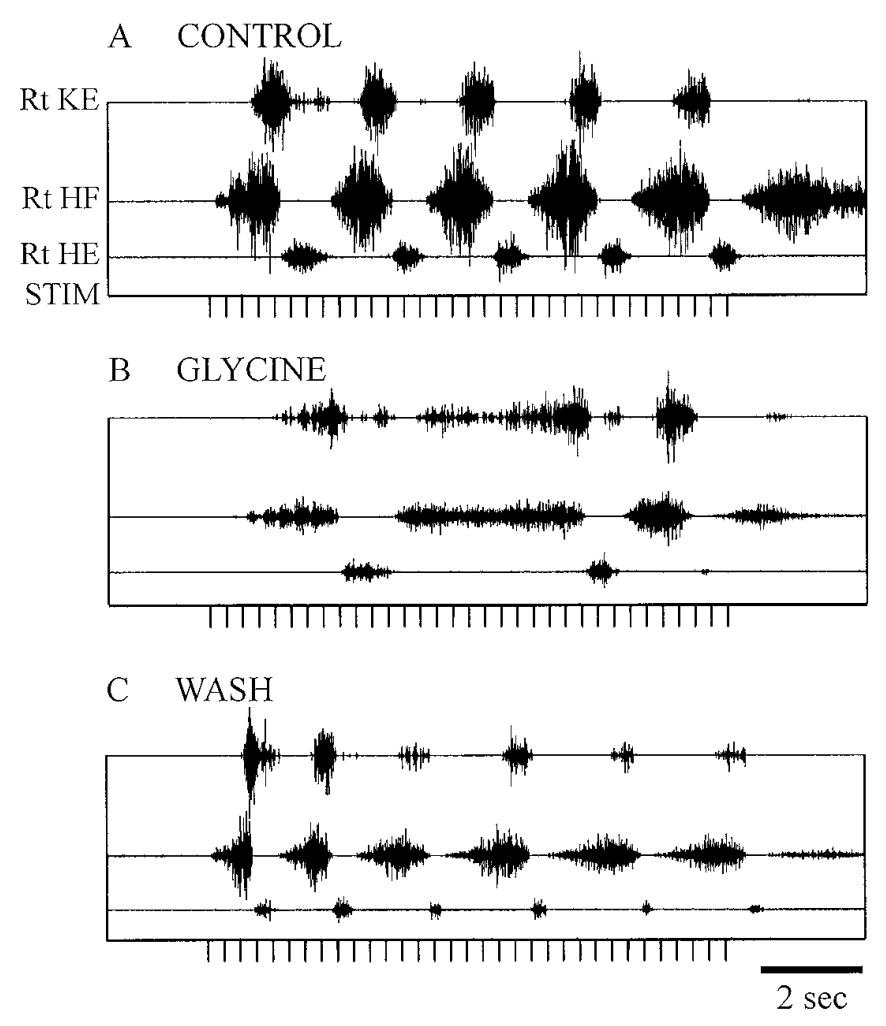

Figure 3. Glycine decreased the frequency and amplitude of rostral scratch ENG bursts. Motor output was recorded from the KE, HF, and HE nerves on the right side in Experiment 8, a D3-end preparation. Stimulation consisted of trains of 33 electrical pulses ( $10 \mathrm{~V}, 1$ msec pulses) delivered at $320 \mathrm{msec}$ interpulse intervals to a site (SP2.5) in the right rostral scratch receptive field. $A$, Control response obtained while spinal segments D7-D10 were bathed in normal saline. $B$, After 35 min superfusion of the exposed spinal segments with $2 \mathrm{~mm}$ glycine. $C$, After $5 \mathrm{hr}+$ 45 min wash with normal saline.

electrical stimulation was applied, pin electrodes were inserted into mirror-image sites (SP2 or SP2.5) (Mortin and Stein, 1990) in the right and left rostral scratch receptive fields; during bilateral stimulation, identical trains of synchronized pulses were delivered to both sides. Unilateral and bilateral stimulus trains were applied in the following sequence: right, left, bilateral, bilateral, right, left (Stein et al., 1995).

\section{Drug application to the spinal cord}

Each experiment consisted of a control, a test, and a wash period. In the first two preparations (Experiments 1 and 2), solutions were applied by pipette onto the exposed spinal cord segments so that the solution partially filled the wax well surrounding the exposed region; in these preparations, the bathing solutions were not stirred or circulated over the cord. In the other twelve preparations (Experiments 3-14), solutions were constantly superfused over the exposed cord at a rate of $5 \mathrm{ml} / \mathrm{min}$ to facilitate diffusional exchange between the solution and the spinal cord tissue. The superfusate was recirculated between the spinal cord and a 10 or $15 \mathrm{ml}$ reservoir for drug solutions (test periods) and a $200 \mathrm{ml}$ reservoir for physiological saline (control and wash periods), using a peristaltic pump (Gilson, Middleton, WI). During the control and wash periods of each experiment, we applied Tris-buffered physiological saline, $\mathrm{pH} 7.4$ (Stein and Schild, 1989), onto the exposed spinal cord segments. During test periods, we applied strychnine hemisulfate (5-50 $\mu \mathrm{M}$ ) or glycine (2 or $5 \mathrm{~mm}$; Sigma, St. Louis, MO) dissolved in physiological saline. Test values of burst frequency (Table 1 ) and onset slopes of integrated HF bursts (Table 2) were obtained after at least $30 \mathrm{~min}$ of strychnine superfusion; wash values were obtained after at least $60 \mathrm{~min}$ of normal saline superfusion.

\section{Data analysis}

Rostral scratch burst frequency. For determination of rostral scratch burst frequency (Table 1), ipsilateral HF ENG recordings and a stimulus marker were digitized off-line at $2 \mathrm{kHz}$ on a $\mathrm{PC}$ and imported into the 


\begin{tabular}{|c|c|c|c|c|c|}
\hline Experiment & $\begin{array}{l}\text { Cord } \\
\text { exposure }\end{array}$ & Stimulus & $\begin{array}{l}\text { Control } \\
\text { average (SD) }\end{array}$ & $\begin{array}{l}\text { Strychnine } \\
\text { average (SD) }\end{array}$ & $\begin{array}{l}\text { Wash } \\
\text { average (SD) }\end{array}$ \\
\hline 1 & D7-D8 & Right & $100.0(51.0)$ & $165.0(31.2)^{\S}$ & $139.9(53.0)^{\mathrm{NS}}$ \\
\hline 1 & D7-D8 & Left & $100.0(13.1)$ & $193.0(27.8)^{*}$ & $176.7(41.4)^{\mathrm{NS}}$ \\
\hline 2 & D7-D10 & Right & $100.0(1.6)$ & $150.0(85.3)^{*}$ & $82.2(15.1)^{*}$ \\
\hline 2 & D7-D10 & Left & $100.0(29.9)$ & $143.5(25.4)^{*}$ & $136.7(7.3)^{\S}$ \\
\hline 8 & D7-D10 & Right & $100.0(23.7)$ & $98.6(26.4)^{\mathrm{NS}}$ & $70.8(27.4)^{*}$ \\
\hline 9 & D7-D10 & Right & $100.0(16.5)$ & $133.1(49.1)^{\ddagger}$ & $86.1(12.5)^{\ddagger}$ \\
\hline 10 & D7-D10 & Right & $100.0(16.0)$ & $93.0(24.9)^{\mathrm{NS}}$ & $94.9(15.9)^{\mathrm{NS}}$ \\
\hline 10 & D7-D10 & Left & $100.0(5.8)$ & $96.4(29.3)^{\mathrm{NS}}$ & $90.2(9.0)^{\mathrm{NS}}$ \\
\hline 11 & D7-D10 & Right & $100.0(20.8)$ & $125.9(48.5)^{\mathrm{NS}}$ & $68.6(45.3)^{*}$ \\
\hline
\end{tabular}

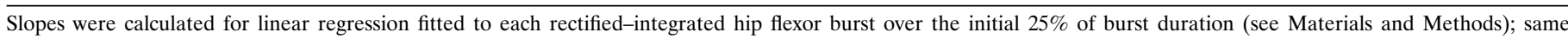

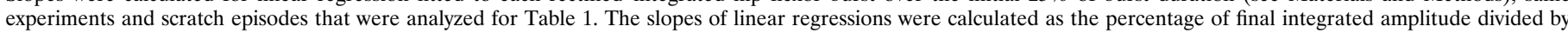

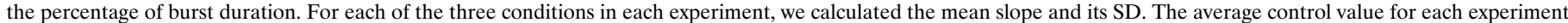
was then set at $100 \%$, and other values in each experiment were expressed as percentages of their respective controls. ${ }^{*} p<0.001 ;{ }^{\circledR} p<0.005 ;{ }^{\ddagger} p<0.03$; ${ }^{\mathrm{NS}} p>0.05$.

waveform processing-analysis program (Run Technologies). Digitized recordings were full wave-rectified and then "rebinned" at $100 \mathrm{~Hz}$, i.e., the mean of 20 consecutive data points was calculated, so that there were 100 full wave-rectified data points per second. The onsets and offsets of HF bursts were identified by the analysis program as positive- and negative-slope crossings over a user-specified voltage threshold. Burst frequencies were automatically calculated as the reciprocal of cycle period, measured between consecutive burst onsets. All analyses were applied only to scratch cycles that occurred completely during the period of stimulation.

Rectified-smoothed and rectified-integrated ENG recordings. Rectifiedsmoothed ENG data are presented (see Figs. $5 A, 6,8$ ) in which digitized recordings ( $2 \mathrm{kHz}$ per channel) from one to six hindlimb muscle nerves were full wave-rectified, rebinned at $100 \mathrm{~Hz}$ (see above), and then smoothed by calculating an even-weighted moving average of each waveform with a 50 $\mathrm{msec}$ bin width. For rectified-integrated data (see Fig. 5A,C), cumulative integrations were calculated for rectified HF nerve recordings over the duration of each rostral scratch burst. In Figure $5 D$, "double-normalized" averages (normalized for amplitude and duration) were calculated for the rectified-integrated HF bursts shown in Figure $5 A$ (first five cycles included in control average; first six cycles included in strychnine average).

Onset slopes of integrated HF bursts. To quantify the onset slopes of rectified-integrated HF ENG bursts (Table 2), we computer-fitted linear regressions to each integrated burst over the initial $25 \%$ of burst duration. The slopes of these regressions were calculated as the percentage of final integrated amplitude divided by the percentage of burst duration. For each experiment, we calculated the mean slope in controls, strychnine, and wash; mean strychnine and wash measurements were expressed as percentages of their respective mean control values.

Phase analysis. We used dual-referent phase measurements to calculate the phase of left HF bursts relative to the activity cycle of the right HF during bilateral rostral scratch motor patterns (see Fig. 7). Dual-referent phase measurements are appropriate for periodic events with a variable duty cycle (Berkowitz and Stein, 1994b). Circular statistics (Batschelet, 1981) were used to analyze phase measurements. The angle of the mean vector in radians divided by $2 \pi$ was the mean phase (expressed on a scale of $0.0-1.0$ ). The length of the mean vector was used in the Rayleigh test to determine the statistical significance of the vector (Batschelet, 1981). The onsets of right HF bursts were defined by phase values of 0.0 and 1.0; offsets were defined by a phase value of 0.5 . The mean vector and angular deviation (= circular analog of SD) were obtained for each set of phase measurements using vector addition (Batschelet, 1981; Berkowitz and Stein, 1994b; Stein et al., 1995). We used the Watson $U^{2}$ test (Batschelet, 1981) to determine whether there was a statistically significant difference between phase values before and after the spinal cord was superfused with strychnine.

\section{RESULTS}

\section{Effects of strychnine and glycine on the burst frequency of fictive rostral scratch motor patterns during unilateral stimulation}

Superfusion of strychnine over spinal segments D7-D8 or D7D10 (in and near the anterior hindlimb enlargement) increased the burst frequency of fictive rostral scratch motor patterns, elicited by electrical or mechanical stimulation of the rostral scratch receptive field on either the right or left side. Experiments were carried out in eleven "D3-end" preparations, two "D3-D10" preparations, and one "D3-D9" preparation. D3-end preparations had intact spinal cords posterior to the D2-D3 transection site; D3-D10 and D3-D9 preparations had complete spinal transections within the drug-superfusion region, at the posterior end of segment D10 or D9, respectively. In the experiment illustrated in Figure 2, we electrically stimulated a site in the left rostral scratch receptive field of a D3-end preparation. Superfusion of $50 \mu \mathrm{M}$ strychnine over the D7-D10 segments increased the frequency of HF ENG bursts during the period of stimulation and decreased burst durations (Fig. 2B), compared with control (Fig. $2 A$ ). These effects were reversed after the spinal cord was washed with normal physiological saline (Fig. 2C).

We quantified the effects of strychnine on HF burst frequencies in six D3-end preparations during rostral scratch motor patterns (Table 1). These preparations were chosen for analysis because they exhibited robust rostral scratch responses on both the right and left sides during electrical shell stimulation under control conditions (before strychnine application). In the present study, we defined a "trial" as a series of stimulations on one side (right or left) for each of the three conditions (control, strychnine, and wash) in a given experiment. In three D3-end preparations (Experiments 8,9 , and 11), left-side trials were not included in the analysis because of frequent "HE deletion" cycles after strychnine superfusion, making it impossible to identify clear HF burst onsets. HE deletions (formerly termed "B-phase deletions") are scratch cycles that exhibit consecutive HF bursts without clear quiescent periods separating them and without corresponding HE bursts (Robertson et al., 1985; Stein et al., 1995). Table 1 shows that treatment with $50 \mu \mathrm{M}$ strychnine produced a statistically significant increase in rostral scratch burst frequency in 7 of the 9 remaining trials, ranging from 118.2 to $194.7 \%$ relative to control averages. A comparable increase in burst frequency was also observed in D3-D10 and D3-D9 preparations during unilateral stimulation (data not shown). Two of these preparations (Experiments 12 and 14) exhibited a reversible increase in burst frequency without HE deletions or other signs of a breakdown in the motor pattern, even after $\geq 2.5 \mathrm{hr}$ of strychnine superfusion. The remaining D3-D10 preparation (Experiment 13) exhibited an increased burst frequency along with frequent HE deletions. In 
addition, continued exposure to strychnine ( $\geq 2 \mathrm{hr}$ ) in this preparation caused the motor pattern to break down completely.

Our goal in these experiments was to investigate the effects of maximal blockade of spinal glycine receptors on rostral scratch motor patterns; therefore, we did not systematically test the effects of low strychnine concentrations. In our first several experiments, however, we tested a series of concentrations ranging from 5 to $50 \mu \mathrm{M}$. Concentrations of 5-20 $\mu \mathrm{M}$ had little or no visible effect on rostral scratch motor patterns, even after 1-2 hr superfusion. In contrast, $50 \mu \mathrm{M}$ strychnine produced obvious effects in most preparations within $20 \mathrm{~min}$. Previous work in our laboratory suggested that $50 \mu \mathrm{M}$ strychnine, superfused over the turtle spinal cord in vivo, blocked glycine receptors but not GABA receptors (Currie and Lee, 1996). In this earlier study, pretreatment of the cord with $50 \mu \mathrm{M}$ strychnine did not prevent exogenous GABA (2 $\mathrm{mM}$ ) from reducing the integrated ENG amplitudes of fictive flexion reflexes, but it did prevent exogenous glycine ( 2 or $5 \mathrm{~mm}$ ) from reducing flexion reflex amplitudes. Strychnine concentrations of up to $50 \mu \mathrm{M}$ were also found to significantly reduce glycine- but not GABA-induced depolarizations in motor neurons of hemisected neonatal rat spinal cords in vitro (Wu et al., 1992) (L. Ziskind-Conhaim, personal communication). Nevertheless, the low sensitivity of our preparations to strychnine calls for caution, because it is known that smaller concentrations (1-10 $\mu \mathrm{M})$ are capable of blocking various ion channels in isolated cell preparations (Shapiro et al., 1974; Oyama et al., 1988) and in the exposed spinal cords of Xenopus embryos (Dale, 1995). The relatively high strychnine concentration required in our experiments may be caused by several factors, including (1) the diffusion barrier presented by the thickness of the spinal hindlimb enlargement (3-4 mm diameter), (2) the intact vascular circulation within the exposed spinal segments, and (3) the fact that only the dorsal surface of the cord was stripped of pial meninges and in direct contact with superfused drug solutions.

In contrast to strychnine, glycine superfusion over the anterior hindlimb enlargement reduced rostral scratch burst frequencies relative to controls. Although we did not routinely compare the effects of strychnine and glycine, in two D3-end preparations we found that glycine superfusion over the D7-D10 spinal segments reversibly lowered burst frequency. In these experiments, mechanical (Experiment 7) or electrical (Experiment 8) stimulation of the right SP2 site elicited control rostral scratch motor patterns with an average HF burst frequency of $0.48 \mathrm{~Hz}$. The HF frequencies after glycine superfusion changed as follows (percentage of control average \pm SD): Experiment 7: (control) $100.0 \pm 8.8$, (glycine, $5 \mathrm{~mm}$ ) $38.7 \pm 25.7$, (wash) $90.1 \pm 18.4$; Experiment 8: (control) $100.0 \pm 11.8$, (glycine, $2 \mathrm{~mm}$ ) $51.8 \pm 13.5$, (wash) $103.5 \pm 9.9$ (five to seven episodes in each condition). Figure 3 shows the effect of glycine on fictive rostral scratch motor patterns in Experiment 8, which was reversed after the spinal cord was washed with normal physiological saline. Glycine effects and recoveries after washing were significant in both experiments at $p<0.005$, using the Mann-Whitney $U$ test.

\section{Effects of strychnine on the amplitude of contralateral ENG bursts during unilateral stimulation}

Strychnine increased the amplitude of contralateral motor output during unilaterally evoked fictive rostral scratch motor patterns. During control responses, we observed low-amplitude ENG activity in contralateral hindlimb nerves alternating with ipsilateral ENG bursts, similar to what has been described previously for the rostral and pocket scratch in turtles (Currie and Stein, 1989;
Berkowitz and Stein, 1994a,b; Stein et al., 1995). Figure $2 A$ shows a typical example of contralateral motor output before strychnine. Stimulation of a site in the left rostral scratch receptive field elicited rostral scratch motor output in all three of the left (ipsilateral) hindlimb nerves and weak rhythmic discharge in the right (contralateral) HE nerve that alternated with left HE bursts. No activity was observed in the right HF or KE nerves. After strychnine superfusion over spinal segments D7-D10, the amplitudes of right $\mathrm{HE}$ bursts were greatly increased and rhythmic bursting appeared de novo in the right HF nerve (Fig. $2 B$ ). These effects were reversed after the cord was washed for several hours with normal saline (Fig. 2C). Note the appearance of three "contralateral HF (cHF) deletion" cycles during strychnine treatment in Figure $2 B$. We define cHF deletions (not described previously) as scratch cycles that exhibit consecutive HE bursts contralateral to the stimulus, without clear quiescent periods separating them and without associated contralateral HF bursts. It is particularly interesting that these $\mathrm{cHF}$ deletions occurred without corresponding ipsilateral HE deletions. This suggests a dissociation of the network components that activate ipsilateral $\mathrm{HE}$ and contralateral HF motor pools, which otherwise appeared to be intimately coupled. The remaining cycles in Figure $2 B$ exhibited 1:1 alternation between right and left $\mathrm{HE}$ discharge and between right and left HF discharge. We observed cHF deletions in this one experiment only; other preparations consistently exhibited 1:1 alternation of right and left hip agonists after strychnine treatment.

In all 11 D3-end preparations, strychnine produced either a de novo appearance $(n=5)$ or increased amplitude $(n=6)$ of contralateral HF discharge during unilateral stimulation. The increased amplitude of contralateral $\mathrm{HE}$ activity was much less reliable, however, being obvious in only two preparations (both with a D7-D10 strychnine superfusion). A possible explanation for this result may be that HF motor neurons have cell bodies located in spinal segments D8 and D9, entirely within the D7-D10 drug-soak region $(n=3)$ and partly within the D7-D8 drug-soak region $(n=8)$. In contrast, the cell bodies of HE motor neurons are located mainly in segments D10-S2 (Ruigrok and Crowe, 1984), largely outside of even the D7-D10 drug-soak region.

\section{Strychnine-induced paroxysmal (seizure) activity}

All of the turtles in this study exhibited some episodic seizure-like motor discharge after prolonged application $(>60 \mathrm{~min})$ of strychnine to the spinal hindlimb enlargement. Although this activity was generally weak and intermittent, it displayed a tendency to increase in frequency and amplitude with prolonged strychnine exposure. In no case did it become so frequent and intense that it interfered with the observation of fictive scratch responses. The shape, amplitude, and frequency of seizure bursts varied considerably across and within experiments, but always began with activity in the right or left HF motor pool. Figure 4 shows an episode of several seizure bursts in one animal. Weak discharges were often confined to the HF motor pool on one side (e.g., Fig. $4 A$, bursts 1 and 2), whereas stronger activity spread from the HF to other motor pools on the same side (data not shown), and the most intense discharges were bilateral, spreading rapidly from one side to the other in either direction (Fig. $4 A$, bursts 3 and 4, B, C). A few preparations $(n=3)$ exhibited rhythmic, mainly bilateral discharges after $>2 \mathrm{hr}$ of strychnine exposure; this activity superficially resembled the strychnine-induced bilateral motor activity that has been observed in neonatal mouse (Droge and Tao, 1993) and rat (Cowley and Schmidt, 1995) spinal cords in vitro. 

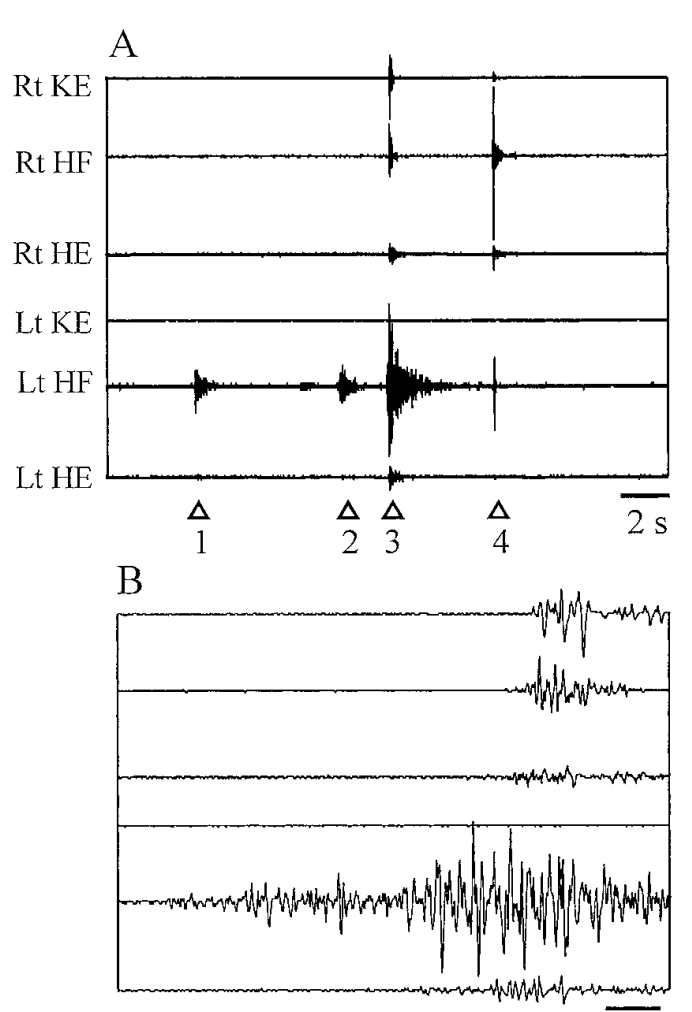

$20 \mathrm{~ms}$

C

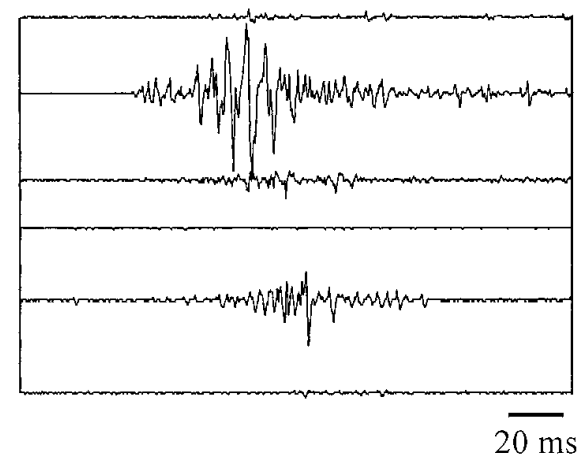

Figure 4. Seizure discharges observed after prolonged exposure to strychnine. Bilateral recordings were obtained from the KE, HF, and HE nerves after spinal segments D7-D10 were superfused with $50 \mu \mathrm{M}$ strychnine for $68 \mathrm{~min}$ in Experiment 6. A, Seizure bursts (unfilled triangles, numbered 1-4). $B$, Time-expansion of burst 3 from $A$, showing left-to-right spread of motor activity. $C$, Time-expansion of burst 4 from $A$, showing right-to-left spread of motor activity.

\section{Effect of strychnine on the shape of rostral scratch HF bursts}

The shape of the observed HF ENG bursts during the fictive rostral scratch was typically fusiform (i.e., spindle-shaped) and similar to results that have been described previously (Robertson and Stein, 1988). At the beginning of the HF burst, only motor neurons with small extracellular action potentials were recruited and there was low-amplitude ENG activity. Motor neurons with medium-sized extracellular action potentials were recruited later in the burst, with the largest HF motor neurons recruited just after $50 \%$ of the burst duration. At the end of the burst, there was an orderly derecruitment of motor neurons, with the smallest motor neurons shutting off last. In the present experiments, we found that strychnine treatment caused the onsets of rostral scratch HF bursts to become more abrupt in some preparations. This effect is illustrated in Figure $5 A, B$, which shows raw and processed recordings of rostral scratch motor output from the ipsilateral HF nerve in Experiment 1, before and after strychnine was applied to spinal segments D7-D8. In Figure $5 C$, we superimposed the second bursts from Figure 5, $A$ and $B$, and normalized them to the same duration to show that HF motor neurons with medium-to-large extracellular spikes were recruited earlier in the burst after strychnine treatment. Similar results were obtained when other bursts from this experiment or other experiments were compared before and after strychnine (data not shown). These results suggest that the onsets of HF ENG bursts became more abrupt after strychnine, because larger units were recruited earlier in the burst. This result is consistent with intracellular recordings, which showed that some HF motor neurons receive synaptic inhibition during the early part of the HF ENG burst (on-cycle inhibition) that overrides an underlying excitatory drive and delays their firing onsets (Robertson and Stein, 1988). Our data suggest that this on-cycle inhibition is at least partly glycinergic.

Figure $5 D$ shows double-normalized averages of the rectifiedintegrated HF ENG bursts from the scratch episodes shown in $A$ and $B$ during the period of stimulation. Strychnine increased the average slope of integrated bursts over the initial 25-30\% of burst duration. We used double-normalized slopes to quantify changes in the shape of ENG bursts so that the results would be independent of burst duration and amplitude. In Table 2, we quantified changes in slope for six different preparations (same trials used in Table 1) by calculating the average double-normalized linear regressions for the first $25 \%$ of burst duration in control, strychnine, and wash conditions (see Materials and Methods). Strychnine produced a statistically significant increase in the average slope for five of nine trials, ranging from 133.3 to $193.0 \%$ of control values.

\section{Effects of strychnine on the timing of intralimb flexor and extensor bursts during unilateral stimulation}

Fictive locomotion studies in mammalian preparations have shown that strychnine blockade of spinal glycine receptors can change the coordination between flexor and extensor ENG bursts within a limb from an alternating to a synchronized relationship (Kriellaars et al., 1988; Noga et al., 1993; Cowley and Schmidt , 1995). In the present study, most preparations (10 of 14) continued to express normal alternation between HF and HE ENG activity after strychnine treatment (up to $2.5 \mathrm{hr}$ ). Synchronous bursting of HF and HE nerves was never observed in any preparation; however, four preparations (three D3-end and one D3-D10) exhibited an increased number of HE deletion cycles after strychnine, which in one case progressed over time to a near-complete disruption of the motor pattern (Fig. $6)$. The D3-D10 preparation shown in Figure 6 had strychnine superfused over the terminal D7-D10 segments, which led to an increased burst frequency (Fig. 6A-C) and increased number of $\mathrm{HE}$ deletion cycles over the first $35 \mathrm{~min}$ of treatment (Fig. 6C). By $50 \mathrm{~min}$ (Fig. 6D), the first $10 \mathrm{sec}$ of the episode was mainly tonic discharge in the $\mathrm{HF}$ and KE nerves. After 2.25 hr (Fig. 6E), there was a near-total loss of rhythmicity, with tonic activity in all three nerves. Note that some organized cycles still developed near the end of the responses in Figure $6 D, E$, after periods of tonic or weakly modulated discharge. A time-expansion of this transition from tonic to rhythmic activity is illustrated in Figure $6 F$, showing that once rhythmicity de- 
A CONTROL

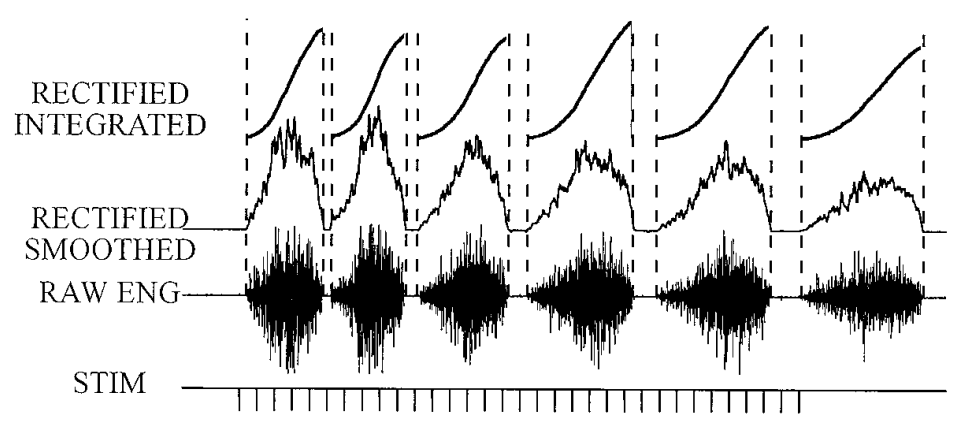

B STRYCHNINE

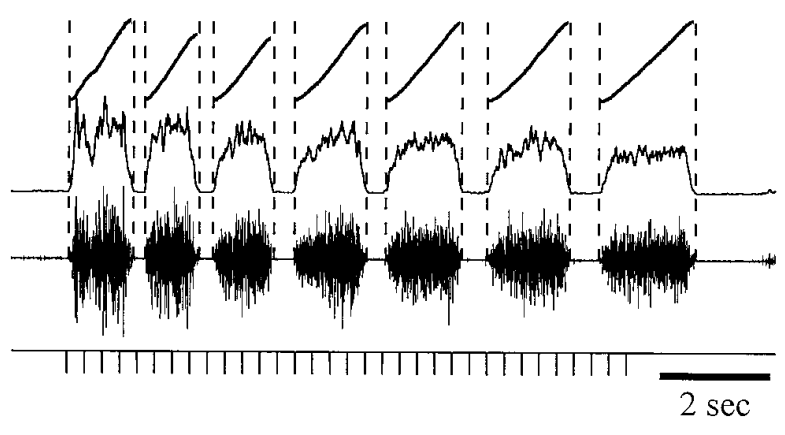

C

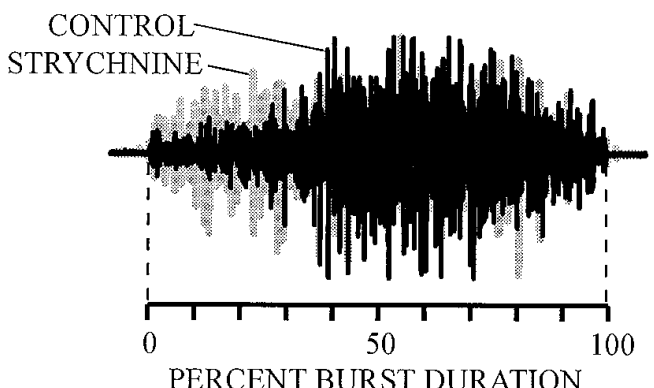

PERCENT BURST DURATION

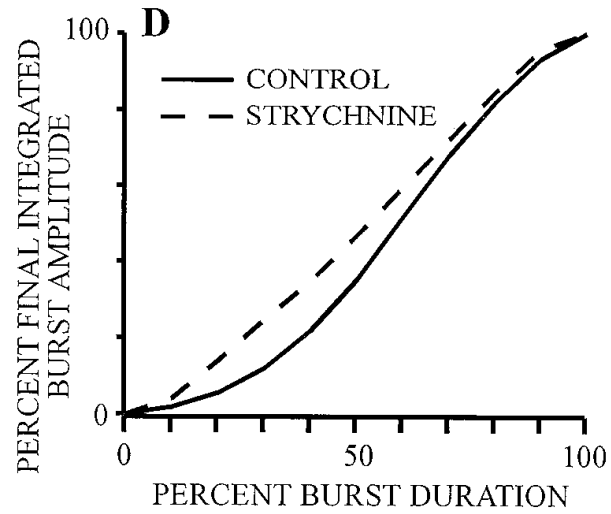

Figure 5. Strychnine changed the shape of HF ENG bursts, producing more abrupt burst onsets and earlier recruitment of medium-large ENG units. Raw and processed ENG recordings from the left HF nerve during electrical stimulation of the SP2 position ( $20 \mathrm{~V}, 1 \mathrm{msec}$ pulses; 33 pulses, $320 \mathrm{msec}$ interpulse intervals) in the left rostral scratch receptive field in Experiment 1. $A$, Response obtained while segments D7-D8 were bathed in normal saline. $B$, After 38 min superfusion with $50 \mu \mathrm{M}$ strychnine. See Materials and Methods for a description of the techniques used for ENG rectification, smoothing, and integration. C, Comparison of CONTROL (black trace) and STRYCHNINE (gray trace) HF bursts from the scratch episodes shown in $A$ and B; the second bursts from both episodes were normalized to the same duration and superimposed. HF motor neurons with larger extracellular spikes were recruited earlier in the burst after strychnine treatment. $D$, Double-normalized averages of rectified-integrated HF bursts from the scratch episodes shown in $A$ and $B$ during the period of stimulation (first five cycles included in CONTROL average; first six cycles included in STRYCHNINE average). Strychnine increased the average slope of integrated bursts over the initial $25-30 \%$ of burst duration. Vertical dashed lines in $A-C$ indicate the timing of burst onsets and burst offsets.

veloped, it still expressed the normal $\mathrm{HF}-\mathrm{HE}$ alternation and the normal KE timing within the hip cycle (compare Fig. 6, $A$ and $F$ ). Thus, in the present experiments, scratch rhythm and pattern generation seemed to be tightly linked and were not clearly separated via widespread blockade of glycinergic transmission.

\section{Effects of strychnine on right-left coordination during bilateral stimulation}

Simultaneous stimulation of sites in the right and left rostral scratch receptive fields produces bilateral rostral scratch motor patterns in which agonist motor pools (e.g., HFs) display alternating activity on the right and left sides (Stein et al., 1995). In the present study, we tested the hypothesis that glycinergic transmission was necessary to maintain an alternating coordination between right and left HF bursts during bilateral scratch motor patterns. Figure 7 shows data from two D3-end preparations, two D3-D10 preparations, and one D3-D9 preparation. We used circular statistics (Batschelet, 1981) to analyze phase measurements. Strychnine produced a highly significant increase in the burst frequency of bilateral scratch motor patterns in all five experiments (Fig. 7A). The effect on interlimb coordination was less obvious, however, because the most consistent observation was an increased variability in the right-left phase relationship (Fig. $7 B$ ). This effect was apparent as larger angular deviations in the average onset and offset phase values of every experiment (left $\mathrm{HF}$ relative to right $\mathrm{HF}$ activity cycle). These increased deviations were largest in D3-D10 and D3-D9 preparations, in which the strychnine had improved access to all of the relevant motor circuitry. Despite the greater variability, however, right and left HF bursts continued to alternate in strychnine, even after $>2 \mathrm{hr}$ of superfusion. In fact, four of five experiments exhibited less overlap between the average left HF burst onset and right HF activity after application of strychnine, whereas three of five experiments also exhibited less overlap between left HF burst offsets and right HF activity.

Figure 8 further illustrates the persistence of right-left alternation in the presence of strychnine. In this D3-D10 preparation (same experiment as shown in Fig. 6), control unilateral stimulation of the right or left side evoked a normal rostral scratch motor pattern in the ipsilateral hindlimb nerves (Fig. 8A1,B1). Unilateral stimulation of the right side also elicited weak bursting from the left (contralateral) HE nerve. Bilateral stimulation in the control elicited brisk rostral scratch motor output from both the right- and left-side hindlimb nerves (Fig. 8C1), with alternating discharge in the right and left HF nerves (Fig. 8D1), similar to what has been described previously (Stein et al., 1995). After $>2$ hr of strychnine superfusion, unilateral stimulation on the right or left side elicited mostly tonic discharge (Fig. 8A2) or disorganized, 


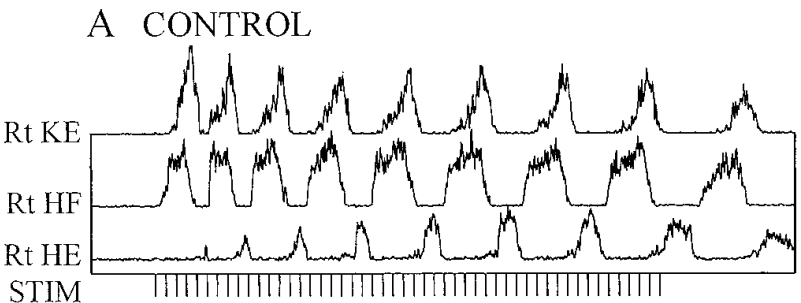

B STRYCHNINE - $20 \mathrm{MIN}$

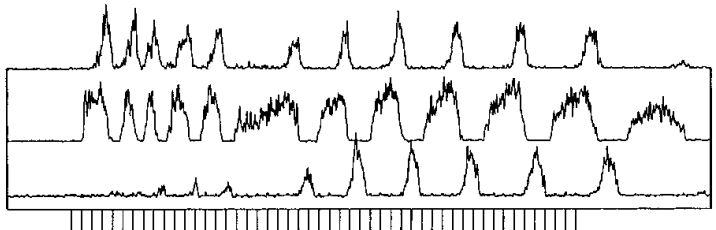

C STRYCHNINE - 35 MIN

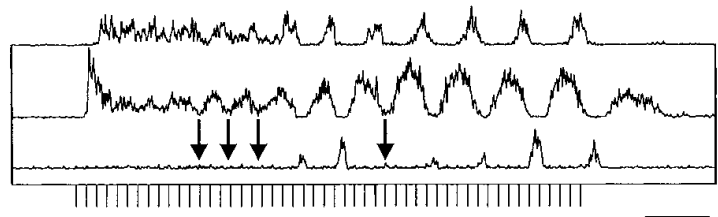

D STRYCHNINE - $50 \mathrm{MIN}$

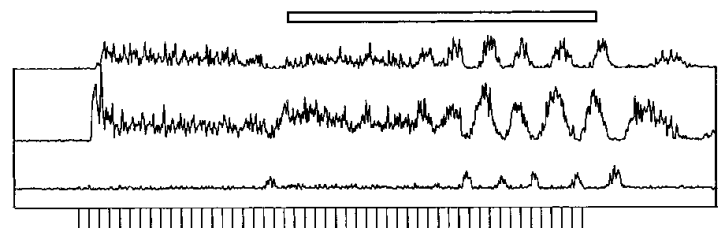

E STRYCHNINE - 2 HRS, 15 MIN

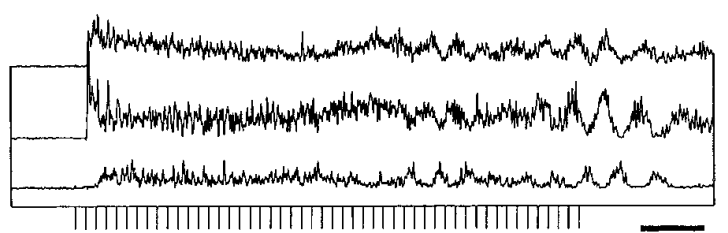

F STRYCHNINE - 50 MIN (EXPANSION)

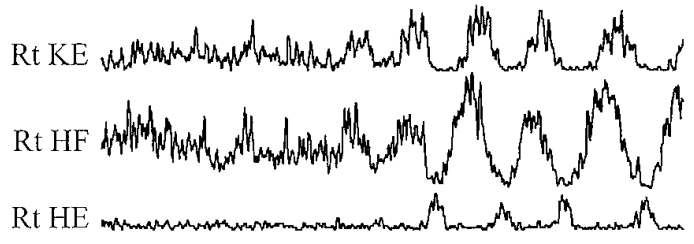

Figure 6. Progressive breakdown in the rostral scratch motor pattern after strychnine application to the spinal cord in a D3-D10 preparation, showing that intralimb phasing remained intact as long as responses remained rhythmic. Fictive rostral scratch motor patterns were recorded from the KE, HF, and $\mathrm{HE}$ nerves on the right side in response to trains of 50 electrical stimulus pulses $(10 \mathrm{~V}, 1 \mathrm{msec}$ pulses, 320 msec interpulse intervals) applied to the SP2 site in the right rostral scratch receptive field in Experiment 13. Recordings were full-wave-rectified, rebinned, and smoothed (see Materials and Methods). Strychnine $(50 \mu \mathrm{M})$ was superfused over spinal segments D7-D10; the spinal cord was transected completely at the posterior end of D10, so that all rostral scratch motor pattern generating circuitry was contained within the drug-soak region. $A$, Response obtained while segments D7-D10 were bathed in normal saline. $B-E$, After $20 \mathrm{~min}-2.25 \mathrm{hr}$ superfusion with $50 \mu \mathrm{M}$ strychnine. Arrows in $C$ indicate the timing of hip extensor (HE) deletions: cycles that lacked ipsilateral HE bursts. $F$, Expansion of the response marked by an unfilled bar in $D$, showing a transition from tonic to rhythmic discharge. ENG amplification in $F$ is twice that in $A-E$. Calibration: 2 sec.

choppy "packets" of activity (Fig. 8B2; also see Fig. 3 in Cowley and Schmidt, 1995) in ipsilateral and contralateral nerves. In contrast, bilateral stimulation in strychnine reestablished rostral scratch rhythmicity on both sides (Fig. 8C2). Bilateral responses still displayed strict alternation between right and left HF bursts (Fig. 8D2). Thus, spinal scratch circuits maintained an alternating right-left coordination even when unilaterally evoked motor patterns were completely disrupted by strychnine. This result provides further support for the hypothesis that alternating interlimb coordination during rostral scratch motor patterns is not critically dependent on glycinergic inhibition.

\section{DISCUSSION}

Glycine is an important "fast" inhibitory transmitter in the vertebrate CNS, particularly in the spinal cord (Aprison, 1990). In the present study, we examined the role of glycinergic transmission within the anterior spinal cord hindlimb enlargement in generating the rhythm and pattern of the hindlimb scratch reflex in turtles. Strychnine, a glycine receptor antagonist (GoodmanGilman et al., 1991), was superfused over two to four adjacent spinal segments while fictive rostral scratch motor output was recorded bilaterally from hindlimb nerves. Strychnine increased the motor burst frequencies (shortened the cycle periods) of unilaterally (Fig. 2, Table 1) and bilaterally evoked (Fig. 7A) rostral scratch motor patterns. Application of glycine had the opposite effect: it reduced burst frequencies (Fig. 3). We are not aware of comparable studies in other scratch reflex preparations; however, a great deal of work has investigated the role of inhibitory transmitters in the spinal control of fictive locomotion. Our results are consistent with fictive locomotion studies in which strychnine consistently increased ventral root burst frequencies (lamprey: Grillner and Wallén, 1980; McPherson et al., 1994; frog embryos: Dale, 1995). In other locomotor work, strychnine produced biphasic effects, either increasing burst frequency without affecting right-left alternation, or decreasing burst frequency while synchronizing the right and left sides (lamprey: Cohen and Harris-Warrick, 1984; Hagevik and McClellan, 1994; neonatal rat: Cowley and Schmidt, 1995). In the present experiments, a high concentration of strychnine $(50 \mu \mathrm{M})$ produced only frequency increases in scratch motor patterns, without significantly affecting right-left alternation (see below).

During the rostral scratch, HF motor neurons are recruited in an orderly fashion according to size, as has been described previously in other systems (Henneman and Mendell, 1981). Motor neurons with small extracellular action potentials are recruited first, followed by cells with medium- and large-sized action potentials. This gradual recruitment of increasingly large units gives rise to the characteristic ramp-like onset of the HF ENG waveform. Robertson and Stein (1988) recorded intracellularly from turtle HF motor neurons during rostral scratch motor patterns. Each of the recorded cells exhibited a characteristic delay between the start of the HF burst and its own firing onset. The authors showed that motor neurons received combined excitatory and inhibitory 

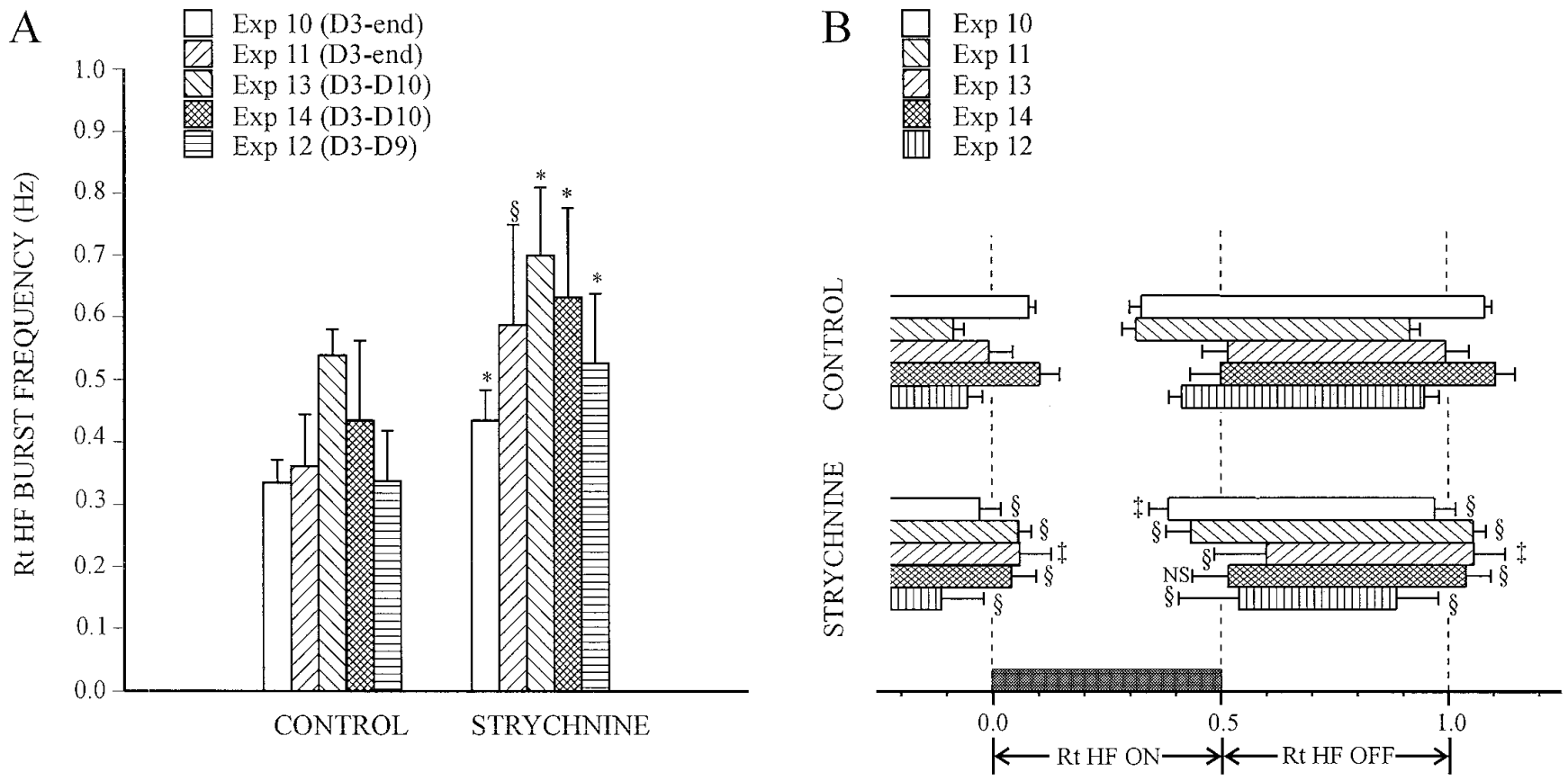

Figure 7. Strychnine $(50 \mu \mathrm{M})$ increased the burst frequencies of bilateral rostral scratch motor patterns $(A)$, but did not synchronize the phase of right and left HF bursts $(B)$ in the same five experiments. $A$, Graphs of rostral scratch burst frequency, comparing $C O N T R O L$ averages obtained while spinal segments D7-D10 were bathed in normal saline, with STRYCHNINE averages obtained after $\geq 30$ min of strychnine superfusion. Burst frequency was measured for right HF bursts during synchronized bilateral electrical stimulation (50 pulses, $320 \mathrm{msec}$ interpulse intervals) of mirror-image sites in the right and left rostral scratch receptive fields. D3-end preparations $($ Exp 10, 11) had intact spinal cords posterior to the D2-D3 transection site; D3-D10 $(\operatorname{Exp} 13,14)$ and D3-D9 (Exp 12) preparations had complete spinal transections within the drug-soak region, at the posterior end of segment D10 or D9, respectively. $B$, Graphs of the average onset and offset phases of left HF bursts relative to two referents in the right HF cycle, comparing CONTROL and STRYCHNINE values. Rectangles in B represent left HF bursts. The left edge of each rectangle represents the average burst onset; the right edge of each rectangle represents the average burst offset, relative to the right $\mathrm{HF}$ activity cycle. The data used to calculate each onset and offset value in $B$ were significantly different from a random distribution ( $p<0.001$; Rayleigh test; Batschelet, 1981). Vertical error bars in $A$ indicate SDs for burst frequency. Horizontal error bars in $B$ indicate angular deviations (see Materials and Methods) for the phase of burst onset or offset. In $A$, we tested for statistical significance within each experiment (strychnine vs control) using the Mann-Whitney $U$ test for linear data (Siegel, 1956). In $B$, we tested for statistical significance for onset and offset phase values (strychnine vs control) using the Watson $\mathrm{U}^{2}$ test for circular data (Batschelet, 1981). ${ }^{*} p<0.0001 ; \S p<0.005$; $\ddagger p<0.05$; NS $p>0.05$.

synaptic drive during this delay, and that the inhibition was sufficiently strong to prevent firing of the later-recruited motor neurons at the start of the HF ENG burst. In the present set of experiments, we showed that blockade of glycine receptors in the spinal segments that contain HF motor neurons caused the onsets of HF ENG bursts to become more abrupt (Fig. 5A, $B$, Table 2); these abnormal burst onsets were characterized by a reduced delay in the firing onset of medium-to-large HF units (Fig. 5C). Cowley and Schmidt (1995) observed a similar shape change in pharmacologically activated locomotor bursts recorded from ankle flexor and extensor nerves in an in vitro preparation of neonatal rat spinal cord. Addition of a glycine antagonist (strychnine) or a $\mathrm{GABA}_{\mathrm{A}}$ antagonist (bicuculline) to the bath solution caused the onsets and offsets of ENG bursts to become more abrupt. Perrins and Soffe (1996) also found that focally applied strychnine lowered the threshold for motor neuron recruitment during fictive swimming in frog embryos. Our data suggest that on-cycle synaptic inhibition of HF motor neurons during the early part of the HF burst (i.e., during the early HF-on phase) is mediated at least partially by strychnine-sensitive glycine receptors. Therefore, glycinergic synaptic inhibition may participate in determining the recruitment timing of $\mathrm{HF}$ motor neurons and the shaping of $\mathrm{HF}$ ENG bursts during the rostral scratch reflex. The fact, however, that four of nine trials did not exhibit a significant change in slope after strychnine application (Table 2) suggests that other mechanisms, such as $\mathrm{GABA}_{\mathrm{A}}$-mediated inhibition, may also contribute.
Strychnine has been reported to synchronize intralimb flexor and extensor nerve discharge during chemically evoked fictive motor patterns in the neonatal rat spinal cord (Cowley and Schmidt, 1995) and during fictive locomotion in cats evoked by electrical stimulation of the mesencephalic locomotor region (Kriellaars et al., 1988; Noga et al., 1993). Intravenous strychnine also abolished the interburst membrane hyperpolarizations in cat spinal motor neurons that normally occurred during excitation of the antagonist motor pool (Pratt and Jordan, 1987). Together, these findings support the concept that glycinergic mechanisms have a role in maintaining flexor-extensor alternation during fictive locomotion.

In the turtle, fictive rostral scratch responses exhibit (1) rhythmic alternation between HF and HE ENG bursts and (2) pure KE (femorotibialis) discharge during the latter part of each HF burst (Robertson et al., 1985). Intracellular recordings revealed chloride-mediated synaptic inhibition in turtle hindlimb motor neurons, which might contribute to both the HF-HE alternation and KE timing during the rostral scratch (Robertson and Stein, 1988). In the present study, we attempted to assess the role of glycinergic inhibition in establishing the timing relationships between intralimb motor pool activities during the rostral scratch. After strychnine treatment, most of our preparations $(n=10$ of 14) continued to express normal alternation between $\mathrm{HF}$ and $\mathrm{HE}$ ENG activity, as well as normal KE timing within the hip cycle (Fig. 2). Several preparations $(n=4)$, however, exhibited an 
CONTROL

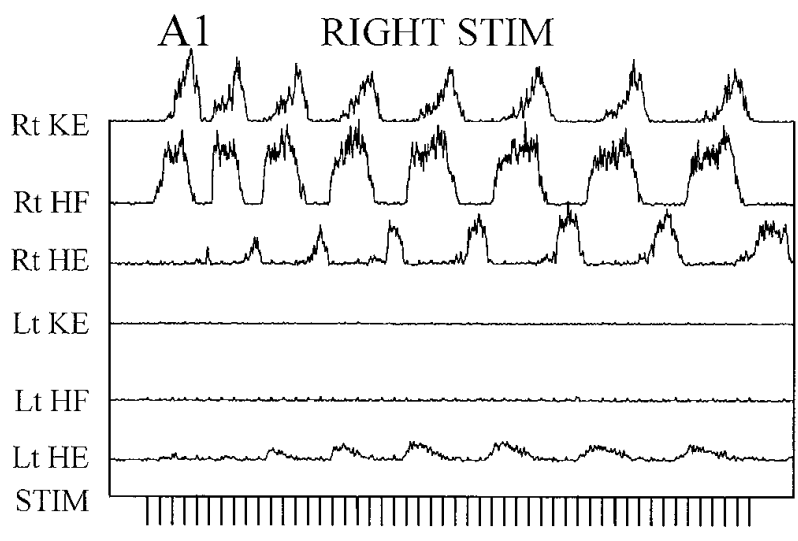

B1

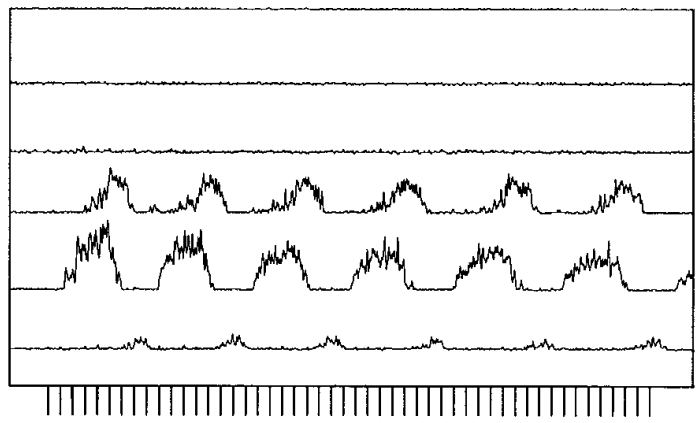

C1
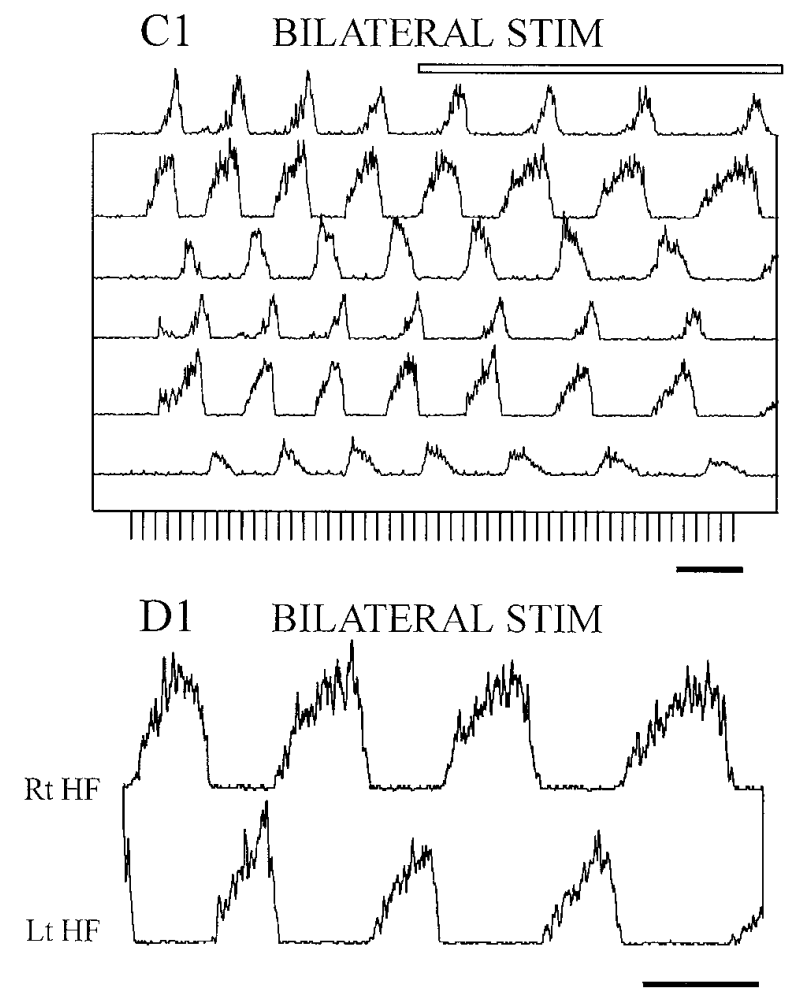

STRYCHININE

A2 RIGHT STIM

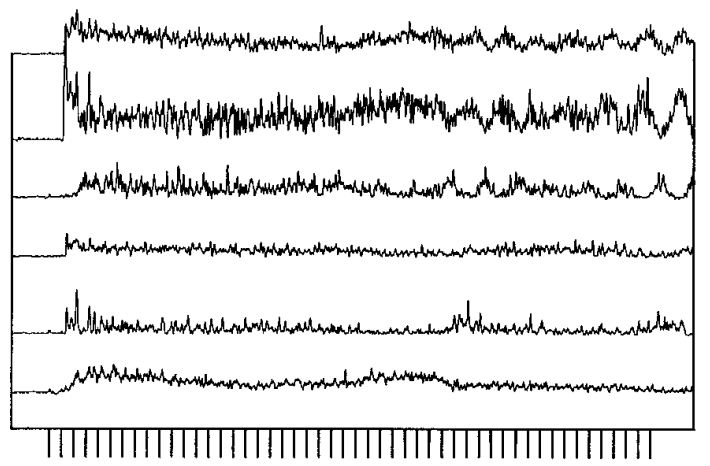

B2

LEFT STIM

C2 BILATERAL STIM

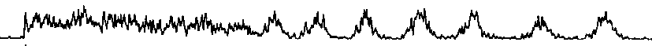

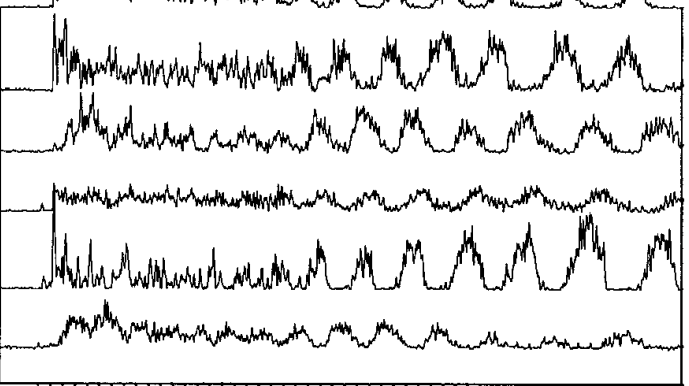

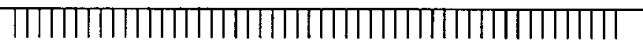

D2 BILATERAL STIM

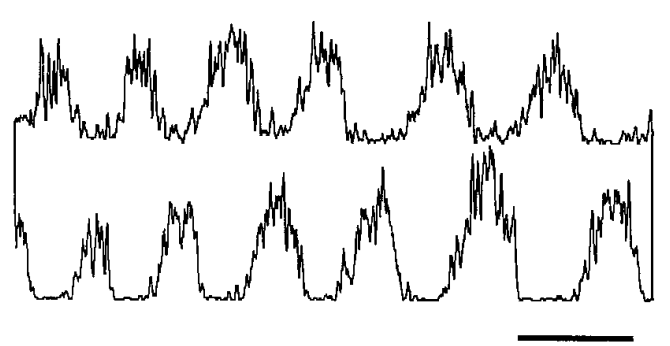

Figure 8. Bilateral stimulation reestablished rostral scratch rhythmicity after prolonged strychnine treatment in Experiment 13 (same preparation as in Fig. 6), which continued to alternate on the right and left sides. Bilateral rectified-smoothed recordings of fictive rostral scratch ENGs obtained from the $\mathrm{KE}, \mathrm{HF}$, and HE nerves while the D7-D10 spinal segments were bathed in normal saline (A1-D1) and after $2.25-2.5$ hr superfusion with 50 $\mu \mathrm{M}$ strychnine $(A 2-D 2)$. Fictive rostral scratch motor patterns were elicited by trains of 50 electrical stimulus pulses $(10 \mathrm{~V}, 1 \mathrm{msec}$ pulses, $320 \mathrm{msec}$ interpulse intervals) applied to the SP2 sites in the rostral scratch receptive fields on the right side $(A 1, A 2)$, the left side $(B 1, B 2)$, and both sides $(C 1, C 2 ; D 1, D 2)$. D1, D2, Expansions of the bilateral scratch motor patterns marked by unfilled bars in $C 1$ and $C 2$, showing only the right and left HF ENGs. ENG amplification in $D 1-D 2$ is twice that in $A 1, A 2-C 1, C 2$. Calibration: $2 \mathrm{sec}$. 
increased number of $H E$ deletion cycles after strychnine, which in one case progressed over time to a near-complete disruption of the motor pattern (Fig. 6). One explanation for an increase in HE deletions is that reduced reciprocal inhibition between ipsilateral HF and HE network "modules" (Stein et al., 1995) may have functionally decoupled $\mathrm{HE}$ activation from the ongoing $\mathrm{HF}$ rhythm. In general, we found that intralimb coordination was disrupted only as the overall scratch rhythm was breaking down. In future studies, it could be useful to apply strychnine via localized microperfusion within the spinal cord (Perrins and Soffe, 1996). Focal drug application combined with single-unit or intracellular recording might allow us to assess the contributions of glycinergic and GABAergic inhibition to the firing pattern of individual hindlimb motor neurons while leaving the overall motor pattern intact.

Unilateral stimulation in the rostral scratch receptive field evokes bilateral motor activity; the contralateral activity, however, is normally much weaker than the ipsilateral motor activity (Fig. 2A) (Stein et al., 1995). Our observation that strychnine increased the amplitude of contralateral motor output during the unilaterally evoked fictive rostral scratch (Fig. $2 B$ ) is consistent with our previous work on the fictive flexion reflex (Currie and Lee, 1996). In that study, strychnine application to the turtle hindlimb enlargement enabled expression of a contralateral (crossed) fictive flexion reflex response to cutaneous stimulation of the foot; these crossed responses were either absent or of extremely low amplitude in controls. Thus, after strychnine treatment, stimulation of the foot on one side elicited bilateral flexion reflex discharge from the right and left HF nerves. A similar crossed flexion reflex was described in cats that had been injected intravenously with strychnine (Çaliskan et al., 1991). Both the flexion reflex work and the present experiments imply the existence of intraspinal glycinergic inhibitory mechanisms that normally mask crossed excitatory pathways and suppress contralateral motor output during cutaneous reflexes.

Our results contrast with fictive locomotion studies in which strychnine was shown to change the right-left coordination from an alternating to a synchronized relationship (lamprey: Cohen and Harris-Warrick, 1984; Alford and Williams, 1989; Hagevik and McClellan, 1994; neonatal rat: Kudo et al., 1991; Cowley and Schmidt, 1995; cat: Noga et al., 1993). We elicited bilateral rostral scratch motor patterns by delivering simultaneous electrical stimulation to mirror-image sites in the right and left rostral scratch receptive fields. Before strychnine treatment, bilateral scratch responses exhibited alternating activity in the right and left $\mathrm{HF}$ motor pools (Figs. 7B, 8D1), as was shown previously (Stein et al., 1995). After strychnine application, the phase-coupling between right and left sides was more variable, but the alternating relationship remained intact (Figs. $7 B, 8 D 2$ ). Synchronized bursting of agonist nerves on the right and left sides was never observed. Our results are consistent with the hypothesis that glycinergic inhibition contributes to the stabilization of right-left coordination during turtle scratch motor patterns but is not key to maintaining the alternating relationship. These data also imply that other mechanisms, such as crossed excitatory connections (Stein et al., 1995), inhibition mediated by $\mathrm{GABA}_{\mathrm{A}}$ receptors (Cowley and Schmidt, 1995), or inhibition mediated by strychnine-insensitive glycine receptors (Kuhse et al., 1990), may have significant roles in right-left coordination during turtle scratch motor patterns.

During unilateral stimulation in the presence of strychnine, ipsilateral HE activity generally alternated $1: 1$ with contralateral $\mathrm{HE}$ activity, further substantiating our conclusion that glycinergic mechanisms are not required to maintain an alternating right-left coordination. In one preparation, however, strychnine application allowed ipsilateral HE bursts to occur without simultaneous inhibition of the contralateral $\mathrm{HE}$ activity and without corresponding cHF bursts (Fig. 2B, arrows). We refer to these cycles as cHF deletions. The occurrence of cHF deletions indicates that under some circumstances the groups of neurons that generate the ipsilateral and contralateral hip rhythms can be functionally decoupled, suggesting that they form separable modules.

The "bilateral shared core" hypothesis of Stein et al. (1995) suggests that a bilaterally distributed subset or "core" of interneurons is shared between the networks that generate the right and left rostral scratch motor patterns. In this model, bilateral stimulation of scratch receptive fields would be expected to excite this shared circuitry to a greater degree than unilateral stimulation. The fact that bilateral stimulation reestablished scratch rhythmicity after strychnine treatment (Fig. 8C,D), after unilaterally evoked responses were completely disrupted, suggests the existence of scratch pattern-generating elements that receive excitatory drive from both sides and thus supports the bilateral shared core hypothesis.

\section{REFERENCES}

Alford S, Williams TL (1989) Endogenous activation of glycine and NMDA receptors in lamprey spinal cord during fictive locomotion. J Neurosci 9:2792-2800.

Aprison MH (1990) The discovery of the transmitter role of glycine. In: Glycine neurotransmission (Ottersen OP, Storm-Mathisen J, eds), pp 1-23. Chichester, UK: Wiley.

Batschelet E (1981) Circular statistics in biology. New York: Academic. Berkowitz A, Stein PSG (1994a) Activity of descending propriospinal axons in the turtle hindlimb enlargement during two forms of fictive scratching: broad tuning to regions of the body surface. J Neurosci 14:5089-5104.

Berkowitz A, Stein PSG (1994b) Activity of descending propriospinal axons in the turtle hindlimb enlargement during two forms of fictive scratching: phase analyses. J Neurosci 14:5105-5119.

Çaliskan S, Tan S, Tan U (1991) Hoffman reflex from foreleg flexor nerves in cats: lateralization, picrotoxin, strychnine, crossed flexor reflex. Int J Neurosci 56:93-106.

Cohen AH, Harris-Warrick RM (1984) Strychnine eliminates alternating motor output during fictive locomotion in the lamprey. Brain Res 293:164-167.

Cowley KC, Schmidt BJ (1995) Effects of inhibitory amino acid antagonists on reciprocal inhibitory interactions during rhythmic motor activity in the in vitro neonatal rat spinal cord. J Neurophysiol 74:1109-1117.

Creed RS, Denny-Brown D, Eccles JC, Liddell EGT, Sherrington CS (1932) Reflex activity of the spinal cord. London: Oxford UP.

Currie SN, Lee S (1995) Effects of strychnine on fictive rostral scratch motor patterns in turtles. Soc Neurosci Abstr 21:153.

Currie SN, Lee S (1996) Glycinergic inhibition in the turtle spinal cord regulates the intensity and pattern of fictive flexion reflex motor output. Neurosci Lett 205:75-78.

Currie SN, Stein PSG (1989) Interruptions of fictive scratch motor rhythms by activation of cutaneous flexion reflex afferents in the turtle. J Neurosci 9:488-496.

Currie SN, Stein PSG (1990) Cutaneous stimulation evokes long-lasting excitation of spinal interneurons in the turtle. $\mathrm{J}$ Neurophysiol 64:1134-1148.

Dale N (1995) Experimentally derived model for the locomotor pattern generator in the Xenopus embryo. J Physiol (Lond) 489:489-510.

Droge MH, Tao Y (1993) Glycine effects on in vitro motor pattern generation in mouse spinal cord. Neurosci Lett 158:139-142.

Goodman-Gilman A, Rall TW, Nies AS, Taylor P (1991) The pharmacological basis of therapeutics. New York: Macmillan.

Grillner S, Wallén P (1980) Does the central pattern generator for locomotion in lamprey depend on glycine inhibition? Acta Physiol Scand 110:103-105.

Hagevik A, McClellan AD (1994) Coupling of spinal locomotor networks in larval lamprey revealed by receptor blockers of inhibitory amino acids: neurophysiology and computer modeling. J Neurophysiol 72:1810-1829. 
Hart BL (1971) Facilitation by strychnine of reflex walking in spinal dogs. Physiol Behav 6:627-628.

Henneman E, Mendell LM (1981) Functional organization of motoneuron pool and its inputs. In: Handbook of physiology, section 1, The nervous system, Vol II, Motor control (Brooks VB, ed), pp 423-508. Bethesda, MD: American Physiological Society.

Kriellaars DJ, Fortier P, Jordan LM (1988) Strychnine-sensitive components of the spinal modules for hindlimb locomotion in the cat. Soc Neurosci Abstr 14:264.

Kudo N, Ozaki S, Yamada T (1991) Ontogeny of rhythmic activity in the spinal cord of the rat. In: Neurobiological basis of human locomotion (Shimamura M, Grillner S, Edgerton VR, eds), pp 127-136. Tokyo: Japan Scientific Societies Press.

Kuhse J, Schmieden V, Betz H (1990) A single amino acid exchange alters the pharmacology of neonatal rat glycine receptor subunit. Neuron 5:867-873.

Marcus LC (1981) Veterinary biology and medicine of captive amphibians and reptiles. Philadephia: Lea and Febiger.

Maxwell JH (1979) Anesthesia and surgery. In: Turtles: perspectives and research (Harless M, Morlock H, eds), pp 127-152. New York: Wiley.

McPherson DR, Buchanan JT, Kasicki S (1994) Effects of strychnine on fictive swimming in the lamprey: evidence for glycinergic inhibition, discrepancies with model predictions, and novel modulatory rhythms. J Comp Physiol [A] 175:311-321.

Mortin LI, Stein PSG (1989) Spinal cord segments containing key elements of the central pattern generators for three forms of scratch reflex in the turtle. J Neurosci 9:2285-2296.

Mortin LI, Stein PSG (1990) Cutaneous dermatomes for initiation of three forms of the scratch reflex in the spinal turtle. J Comp Neurol 295:515-529.

Noga BR, Cowley KC, Huang A, Jordan LM, Schmidt BJ (1993) Effects of inhibitory amino acid antagonists on locomotor rhythm in the decerebrate cat. Soc Neurosci Abstr 19:540.

Oyama Y, Akaike N, Carpenter DO (1988) Strychnine decreases the voltage-dependent $\mathrm{Ca}^{2+}$ current of both Aplysia and frog ganglion neurons. Cell Mol Neurobiol 8:307-314.
Perrins R, Soffe SR (1996) Local effects of glycinergic inhibition in the spinal cord motor systems for swimming in amphibian embryos. J Neurophysiol 76:1025-1035.

Pratt CA, Jordan LM (1987) Ia inhibitory interneurons and Renshaw cells as contributors to the spinal mechanisms for fictive locomotion. J Neurosci 57:56-71.

Robertson GA, Stein PSG (1988) Synaptic control of hindlimb motoneurones during three forms of the fictive scratch reflex in the turtle. J Physiol (Lond) 404:101-128.

Robertson GA, Mortin LI, Keifer J, Stein PSG (1985) Three forms of the scratch reflex in the spinal turtle: central generation of motor patterns. J Neurophysiol 53:1517-1534.

Ruigrok TJH, Crowe A (1984) The organization of motor neurons in the turtle lumbar spinal cord. J Comp Neurol 228:24-37.

Shapiro BI, Wang CM, Narahashi T (1974) Effects of strychnine on ionic conductances of squid giant axon membrane. J Pharmacol Exp Ther 188:66-76.

Siegel S (1956) Nonparametric statistics for the behavioral sciences. New York: McGraw-Hill.

Stein PSG (1989) Spinal cord circuits for motor pattern selection in the turtle. Ann NY Acad Sci 563:1-10.

Stein PSG, Schild CP (1989) $N$-methyl-D-aspartate antagonist applied to the spinal cord hindlimb enlargement reduces the amplitude of flexion reflex in the turtle. Brain Res 479:379-380.

Stein PSG, Victor JC, Field EC, Currie SN (1995) Bilateral control of hindlimb scratching in the spinal turtle: contralateral spinal circuitry contributes to the normal ipsilateral motor pattern of fictive rostral scratching. J Neurosci 15:4343-4355.

Wu W-I, Ziskind-Conhaim L, Sweet MA (1992) Early development of glycine- and GABA-mediated synapses in rat spinal cord. J Neurosci 12:3935-3945.

Zangerl R (1969) The turtle shell. In: Biology of the reptilia, Vol 1 (Gans C, ed), pp 311-339. New York: Academic. 